\title{
The generalized Kupershmidt deformation for constructing new integrable systems from integrable bi-Hamiltonian systems
}

\author{
Yuqin Yad1 and Yunbo Zeng2 \\ 1) Department of Applied Mathematics, China Agricultural University, Beijing, \\ 100083, PR China \\ ${ }^{2)}$ Department of Mathematical Science, Tsinghua University, Beijing, 100084, PR \\ China
}

\begin{abstract}
Based on the Kupershmidt deformation for any integrable bi-Hamiltonian systems presented in [4], we propose the generalized Kupershmidt deformation to construct new systems from integrable bi-Hamiltonian systems, which provides a nonholonomic perturbation of the bi-Hamiltonian systems. The generalized Kupershmidt deformation is conjectured to preserve integrability. The conjecture is verified in a few representative cases: KdV equation, Boussinesq equation, Jaulent-Miodek equation and Camassa-Holm equation. For these specific cases, we present a general procedure to convert the generalized Kupershmidt deformation into the integrable Rosochatius deformation of soliton equation with self-consistent sources, then to transform it into a $t$-type bi-Hamiltonian system. By using this generalized Kupershmidt deformation some new integrable systems are derived. In fact, this generalized Kupershmidt deformation also provides a new method to construct the integrable Rosochatius deformation of soliton equation with self-consistent sources.
\end{abstract}

\footnotetext{
${ }^{1}$ Corresponding author: yqyao@math.tsinghua.edu.cn

2yzeng@math.tsinghua.edu.cn
} 


\section{Introduction}

It is known that one can construct a new integrable system starting from a bi-Hamiltonian system. Fuchssteiner and Fokas showed [1] that compatible symplectic structures lead in natural way to hereditary symmetries, which provides a method to construct a hierarchy of exactly solvable evolution equations. Olver and Rosenau [2] demonstrated that most integrable bi-Hamiltonian systems are governed by a compatible trio of Hamiltonian structures, and their recombination leads to integrable hierarchies of nonlinear equations.

Recently for the following KdV6 equation or nonholonomic deformation of $\mathrm{KdV}$ equation derived in 3

$$
\begin{aligned}
& u_{t}=u_{x x x}+6 u u_{x}-\omega_{x}, \\
& \omega_{x x x}+4 u \omega_{x}+2 u_{x} \omega=0,
\end{aligned}
$$

Kupershmidt found [4 that (11) can be converted into

$$
\begin{aligned}
& u_{t}=B_{1}\left(\frac{\delta H_{3}}{\delta u}\right)-B_{1}(\omega), \\
& B_{2}(\omega)=0,
\end{aligned}
$$

where

$$
B_{1}=\partial=\partial_{x}, B_{2}=\partial^{3}+2(u \partial+\partial u)
$$

are the two standard Hamiltonian operators of the KdV hierarchy and $H_{3}=$

$u^{3}-\frac{u_{x}^{2}}{2}$. In general, for a bi-Hamiltonian system

$$
u_{t_{n}}=B_{1}\left(\frac{\delta H_{n+1}}{\delta u}\right)=B_{2}\left(\frac{\delta H_{n}}{\delta u}\right),
$$

the ansatz (2) provides a nonholonomic deformation of bi-Hamiltonian systems (4):

$$
\begin{gathered}
u_{t_{n}}=B_{1}\left(\frac{\delta H_{n+1}}{\delta u}\right)-B_{1}(\omega), \\
B_{2}(\omega)=0
\end{gathered}
$$


which is called as Kupershmidt deformation of bi-Hamiltonian systems. This deformation is conjectured to preserve integrability and the conjecture is verified in a few representative cases in [4].

In 5, we showed that the Kupershmidt deformation (2) of KdV equation is equivalent to the integrable Rosochatius deformation of $\mathrm{KdV}$ equation with self-consistent sources, and constructed the bi-Hamiltonian structure for the Kupershmidt deformation of KdV equation (2). The conjecture is then proved in [6] that the Kupershmidt deformation of a bi-Hamiltonian system is itself bi-Hamiltonian.

Rosochatius found that it would still keep the integrability to add a potential of the sum of inverse squares of the coordinates to that of the Neumann system [7]. The deformed system is called as Neumann-Rosochatius system. Then the Rosochatius deformation of Garnier system, Jacobi system and many constrained flows of soliton equations were constructed in [8, 9, 10, This Rosochatius-type integrable systems have important physical applications [11, 12, 13. However, these Rosochatius deformations are limited to few finitedimensional integrable Hamiltonian systems(FDIHS). Recently, in 14 we proposed a systematic method for generalizing the integrable Rosochatius deformation for FDIHS to integrable Rosochatius deformation for infinite-dimensional integrable equations. Many integrable Rosochatius deformations of soliton equations with self-consistent sources and their Lax representations were presented in [14, 15].

In present paper, based on the Kupershmidt deformation (5), we propose the generalized Kupershmidt deformation (GKD) to construct new systems from integrable bi-Hamiltonian systems which provides a nonholonomic perturbation of the bi-Hamiltonian systems. The generalized Kupershmidt deformation is conjectured to preserve integrability. Although it is difficult to prove the integrability in general, the conjecture can be verified in many specific cases. Using KdV equation, Boussinesq equation, Jaulent-Miodek equation and Camassa- 
Holm equation as examples, we present a general procedure to show how to convert these generalized Kupershmidt deformations into the Rosochatius deformations of soliton equations with self-consistent sources. These Rosochatius deformations of soliton equations with self-consistent sources possess the Lax representations, which are easy constructed by using the systematic method in [14, 15], and their stationary equations can be shown to be finite-dimensional integrable Hamiltonian systems in the Liouville's sense [14, 15, 16]. Furthermore, for the specific Rosochatius deformations of soliton equations with self-consistent sources there is a general procedure to transform it into a $t$-type bi-Hamiltonian system by introducing the Jacobi-Ostrogradiski coordinates and taking the spacial variable $x$ as the evolution parameter according to $[17,18,19,20,5$. These facts imply the integrability of the Rosochatius deformations of soliton equations with self-consistent sources. Indeed the generalized Kupershmidt deformation also provides a new method for obtaining the Rosochatius deformation of soliton equation with self-consistent sources, which is quite different from the method presented in [14, 15].

In section 2, we propose the the generalized Kupershmidt deformation (GKD) of bi-Hamiltonian system, by using GKD of KdV hierarchy to illustrate the formulae. Section 3 is devoted to a new integrable system obtained from the GKD of Camassa-Holm equation and shows how to transform the GKD of CamassaHolm equation into the integrable Rosochatius deformation of Camassa-Holm equation with self-consistent sources. Section 4 treats the GKD of Boussinesq equation. The last section presents the GKD of Jaulent-Miodek hierarchy, and demonstrate how to convert the GKD of a integrable system into a biHamiltonian system with $t$-type Hamiltonian operator by taking $x$ as evolution parameter and $t$ as 'spatial' variable. 


\section{The generalized Kupershmidt deformation of bi-Hamiltonian systems}

Consider a hierarchy of soliton equations with bi-Hamiltonian structure

$$
u_{t_{n}}=B_{1}\left(\frac{\delta H_{n+1}}{\delta u}\right)=B_{2}\left(\frac{\delta H_{n}}{\delta u}\right),
$$

where $B_{1}$ and $B_{2}$ are two standard Hamiltonian operators. The associated spectral problem reads

$$
L(u) \phi=\lambda \phi
$$

For the eigenvalue $\lambda$, it is easy to find that the variational derivative of $\lambda$

$$
\frac{\delta \lambda}{\delta u}=f(\varphi)
$$

Assume that $\lambda_{j}, j=1, \ldots N$ are $N$ distinct real eigenvalues of (7), we have

$$
L \varphi_{j}=\lambda_{j} \varphi_{j}, j=1,2, \cdots, N
$$

and we denote

$$
\frac{\delta \lambda_{j}}{\delta u}=\left.\frac{\delta \lambda}{\delta u}\right|_{\lambda=\lambda_{j}}=f\left(\varphi_{j}\right)
$$

Based on the Kupershmidt deformation (5), we first generalize Kupershmidt deformation as follows

$$
\begin{aligned}
& u_{t_{n}}=B_{1}\left(\frac{\delta H_{n+1}}{\delta u}\right)-B_{1}\left(\sum_{j=1}^{N} \omega_{j}\right), \\
& \left(B_{2}-\alpha_{j} B_{1}\right)\left(\omega_{j}\right)=0, j=1,2, \cdots, N,
\end{aligned}
$$

where $\alpha_{j}$ are arbitrary constants, which also gives rise to a nonholonomic deformation of bi-Hamiltonian systems (6) similar to the integrable KdV6's type noholonomic deformation of soliton equations (6). So (8) provides a way to construct new systems from the bi-Hamiltonian systems (6). Furthermore, observe that $\omega_{j}$ in (8a) are at the same position as $\frac{\delta H_{n+1}}{\delta u}$, and the eigenvalues $\lambda_{j}$ are also the conserved quantities for (6) as $H_{n}$, it is reasonable to take $\omega_{j}=\frac{\delta \lambda_{j}}{\delta u}$ 
and this setting is compatible with (8b). So we finally propose the generalized Kupershmidt deformation for a bi-Hamiltonian systems as follows

$$
\begin{aligned}
& u_{t_{n}}=B_{1}\left(\frac{\delta H_{n+1}}{\delta u}-\sum_{j=1}^{N} \frac{\delta \lambda_{j}}{\delta u}\right), \\
& \left(B_{2}-\alpha_{j} B_{1}\right)\left(\frac{\delta \lambda_{j}}{\delta u}\right)=0, j=1,2, \cdots, N .
\end{aligned}
$$

As the conjecture made in [4], it is reasonable to conjecture the integrability of the new system (9). It seems that it is difficult to prove the integrability in general. However it can be verified in many specific cases. Using $\mathrm{KdV}$ equation, Boussinesq equation, Jaulent-Miodek equation and Camassa-Holm equation as examples, we will proceed to the general procedure to convert these generalized Kupershmidt deformations into the Rosochatius deformations of soliton equations with self-consistent sources. By using the systematic method in 14, 15, it is easy to construct the Lax representation for these Rosochatius deformations of soliton equations with self-consistent sources, and to show their stationary equations to be finite-dimensional integrable Hamiltonian systems in the Liouville's sense [14, 15]. Furthermore, following the method proposed in [17, 18, 19, 20, we have presented a general procedure to transform the Rosochatius deformations of soliton equations with self-consistent sources into a bi-Hamiltonian system by introducing the Jacobi-Ostrogradiski coordinates and taking the spacial variable $x$ as the evolution parameter in [5. We will use the GKD of Jaulent-Miodek equation to illustrate the general constructure. These facts imply the integrability of the generalized Kupershmidt deformation of soliton equation. So the deformation (8) and (9) provides a way to construct new integrable systems from integrable bi-Hamiltonian systems and to establish the integrable Rosochatius deformation of soliton equation with self-consistent sources in different way from that in 14,15$]$.

We now use the KdV hierarchy to illustrate the procedure. Consider the 
Schrödinger eigenvalue problem

$$
\phi_{x x}+(u-\lambda) \phi=0
$$

the associated KdV hierarchy read

$$
u_{t_{n}}=B_{1}\left(\frac{\delta H_{n+1}}{\delta u}\right)=B_{2}\left(\frac{\delta H_{n}}{\delta u}\right), n=1,2, \cdots
$$

where

$$
\begin{gathered}
B_{1}=\partial=\partial_{x}, \quad B_{2}=\partial^{3}+2(u \partial+\partial u) \\
H_{n+1}=\int b_{n+1} d x, \quad b_{n+1}=-\frac{2}{2 n+1} R^{n} u, \quad R=-\frac{1}{4} \partial^{2}-u+\frac{1}{2} \partial^{-1} u_{x} .
\end{gathered}
$$

It is easy to find that

$$
\frac{\delta \lambda}{\delta u}=\varphi^{2}
$$

For $N$ distinct eigenvalues $\lambda_{j}$, consider the spectral problem

$$
\varphi_{j x x}+\left(u-\lambda_{j}\right) \varphi_{j}=0, j=1,2, \cdots, N
$$

We have

$$
\frac{\delta \lambda_{j}}{\delta u}=\varphi_{j}^{2}
$$

For $n=2, \alpha_{j}=\lambda_{j}$, (8) gives rise to the following new integrable generalized KdV6 equation

$$
\begin{aligned}
& u_{t}=u_{x x x}+6 u u_{x}-\sum_{j=1}^{N} \omega_{j x}, \\
& \omega_{j x x x}+4 u \omega_{j x}+2 u_{x} \omega_{j}-\lambda_{j} \omega_{j x}=0, j=1,2, \cdots, N .
\end{aligned}
$$

(9b) yields

$$
2 \varphi_{j}\left[\varphi_{j x x}+\left(u-\lambda_{j}\right) \varphi_{j}\right]_{x}+6 \varphi_{j x}\left[\varphi_{j x x}+\left(u-\lambda_{j}\right) \varphi_{j}\right]=0,
$$

which immediately gives rise to

$$
\varphi_{j x x}+\left(u-\lambda_{j}\right) \varphi_{j}=\frac{\mu_{j}}{\varphi_{j}^{3}},
$$


where $\mu_{j}, j=1,2, \cdots, N$ are integral constants. When $n=2, \alpha_{j}=\lambda_{j}$,(9) gives rise to the following generalized Kupershmidt deformation of KdV equation

$$
\begin{aligned}
& u_{t}=\frac{1}{4}\left(u_{x x x}+6 u u_{x}\right)-\sum_{j=1}^{N}\left(\varphi_{j}^{2}\right)_{x}, \\
& \varphi_{j x x}+\left(u-\lambda_{j}\right) \varphi_{j}=\frac{\mu_{j}}{\varphi_{j}^{3}}, j=1,2, \cdots, N
\end{aligned}
$$

which is just the integrable Rosochatius deformation of $\mathrm{KdV}$ equation with self-consistent sources (RD-KdVHSCS) presented in [14. When $\mu_{j}=0, j=$ $1, \cdots, N$, 13] reduces to the $\mathrm{KdV}$ equation with self-consistent sources 21. The Lax pair for (13) was constructed by a systematic method in 14 .

$$
\begin{aligned}
& \left(\begin{array}{l}
\psi_{1} \\
\psi_{2}
\end{array}\right)_{x}=U\left(\begin{array}{l}
\psi_{1} \\
\psi_{2}
\end{array}\right), U=\left(\begin{array}{cc}
0 & 1 \\
\lambda-u & 0
\end{array}\right), \\
& \left(\begin{array}{l}
\psi_{1} \\
\psi_{2}
\end{array}\right)_{t}=V\left(\begin{array}{l}
\psi_{1} \\
\psi_{2}
\end{array}\right), \\
& V=\left(\begin{array}{cc}
-\lambda^{2}-\frac{u}{2} \lambda-\frac{u_{x x}}{4}-\frac{u^{2}}{2}+\frac{1}{2} \sum_{j=1}^{N} \varphi_{j}^{2} & \frac{u_{x}}{4}
\end{array}\right) \\
& -\frac{1}{2} \sum_{j=1}^{N} \frac{1}{\lambda-\lambda_{j}}\left(\begin{array}{cc}
\varphi_{j} \varphi_{j x} & -\varphi_{j}^{2} \\
\varphi_{j x}^{2}+\frac{\mu_{j}}{\varphi_{j}^{2}} & -\varphi_{j} \varphi_{j x}
\end{array}\right) .
\end{aligned}
$$

The stationary equation of (13) reduces to the generalized integrable HénonHeiles system [14]. The bi-Hamiltonian structure for (13) was presented in [5]

\section{The new integrable system obtained from the Camassa-Holm equation}

The Camassa-Holm (CH) equation [1, 22, 23] read

$$
m_{t}=B_{1} \frac{\delta H_{1}}{\delta u}=B_{2} \frac{\delta H_{0}}{\delta u}=-2 u_{x} m-u m_{x}, \quad m=u-u_{x x}+\omega
$$


where

$$
B_{1}=-\partial+\partial^{3}, B_{2}=m \partial+\partial m
$$

are the two standard Hamiltonian operators of the $\mathrm{CH}$ equation and

$$
H_{0}=\frac{1}{2} \int\left(u^{2}+u_{x}^{2}\right) d x, H_{1}=\frac{1}{2} \int\left(u^{3}+u u_{x}^{2}\right) d x .
$$

The Lax pair for $\mathrm{CH}$ equation is

$$
\begin{aligned}
& \phi_{x x}=\left(\frac{1}{4}-\frac{1}{2} m \lambda\right) \phi, \\
& \phi_{t}=\frac{1}{2} u_{x} \phi-\left(\frac{1}{\lambda}+u\right) \phi_{x} .
\end{aligned}
$$

For $N$ distinct real eigenvalues $\lambda_{j}$, consider the following spectral problem

$$
\varphi_{j x x}=\frac{1}{4} \varphi_{j}-\frac{1}{2} m \lambda_{j} \varphi_{j}, j=1,2, \cdots, N .
$$

It is easy to find that

$$
\frac{\delta \lambda}{\delta m}=\lambda \varphi^{2}, \quad \frac{\delta \lambda_{j}}{\delta m}=\lambda_{j} \varphi_{j}^{2} .
$$

Then we obtain the following new nonholonomic deformation of the CamassaHolm equation from (8) with $\alpha_{j}=\frac{1}{\lambda_{j}}$

$$
\begin{aligned}
& m_{t}=-2 u_{x} m-u m_{x}+\sum_{j=1}^{N}\left[\omega_{j x}-\omega_{j x x x}\right], \\
& 2 m \omega_{j x}+m_{x} \omega_{j}+\lambda_{j}\left[\omega_{j x}-\omega_{j x x x}\right]=0, j=1,2, \cdots, N .
\end{aligned}
$$

Take $\alpha_{j}=\frac{1}{\lambda_{j}}$, Eq. (9) leads to the following generalized Kupershmidt deformation of $\mathrm{CH}$

$$
\begin{aligned}
& m_{t}=B_{1}\left(\frac{\delta H_{1}}{\delta m}-\sum_{j=1}^{N} \frac{1}{\lambda_{j}} \frac{\delta \lambda_{j}}{\delta m}\right)=-2 u_{x} m-u m_{x}+\sum_{j=1}^{N}\left[\left(\varphi_{j}^{2}\right)_{x}-\left(\varphi_{j}^{2}\right)_{x x x}\right], \\
& \left(B_{2}-\frac{1}{\lambda_{j}} B_{1}\right)\left(\frac{1}{\lambda_{j}} \frac{\delta \lambda_{j}}{\delta m}\right)=0, j=1,2, \cdots, N .
\end{aligned}
$$

Then (19b) yields

$$
2 \varphi_{j}\left(\varphi_{j x x}+\frac{1}{2} \lambda_{j} m \varphi_{j}-\frac{1}{4} \varphi_{j}\right)_{x}+6 \varphi_{j x}\left(\varphi_{j x x}+\frac{1}{2} \lambda_{j} m \varphi_{j}-\frac{1}{4} \varphi_{j}\right)=0
$$


which immediately gives rise to

$$
\varphi_{j x x}=\frac{1}{4} \varphi_{j}-\frac{1}{2} m \lambda_{j} \varphi_{j}+\frac{\mu_{j}}{\varphi_{j}^{3}} .
$$

So Eq. (19) gives a new integrable system

$$
\begin{aligned}
& m_{t}=-2 u_{x} m-u m_{x}+\sum_{j=1}^{N}\left[\left(\varphi_{j}^{2}\right)_{x}-\left(\varphi_{j}^{2}\right)_{x x x}\right], \\
& \varphi_{j x x}=\frac{1}{4} \varphi_{j}-\frac{1}{2} m \lambda_{j} \varphi_{j}+\frac{\mu_{j}}{\varphi_{j}^{3}}, j=1,2, \cdots, N
\end{aligned}
$$

which lax pair can be found by using the method in 24

$$
\begin{aligned}
& \left(\begin{array}{l}
\psi_{1} \\
\psi_{2}
\end{array}\right)_{x}=U\left(\begin{array}{l}
\psi_{1} \\
\psi_{2}
\end{array}\right), U=\left(\begin{array}{cc}
0 & 1 \\
\frac{1}{4}-\frac{1}{2} \lambda m & 0
\end{array}\right) \\
& \left(\begin{array}{l}
\psi_{1} \\
\psi_{2}
\end{array}\right)_{t}=V\left(\begin{array}{l}
\psi_{1} \\
\psi_{2}
\end{array}\right), \\
& V=\left(\begin{array}{cc}
\frac{u_{x}}{2} & -\frac{1}{\lambda}-u \\
\frac{u}{4}-\frac{1}{4 \lambda}+\frac{m u \lambda}{2} & -\frac{u_{x}}{2}
\end{array}\right) \\
& -\sum_{j=1}^{N} \frac{\lambda \lambda_{j}}{\lambda-\lambda_{j}}\left(\begin{array}{cc}
\varphi_{j} \varphi_{j x} & -\varphi_{j}^{2} \\
\varphi_{j x}^{2}+\frac{\mu_{j}}{\varphi_{j}^{2}} & -\varphi_{j} \varphi_{j x}
\end{array}\right)
\end{aligned}
$$

In fact Eq. (20) can also be regarded as the RD-CHESCS.

\section{The generalized Kupershmidt deformation of Boussinesq equation}

For the following third-order eigenvalue problem 25]

$$
L \phi=\phi_{x x x}+v \phi_{x}+\left(\frac{1}{2} v_{x}+w\right) \phi=\lambda \phi,
$$

the associated Boussinesq equation is

$$
\left(\begin{array}{c}
v \\
w
\end{array}\right)_{t}=B_{1}\left(\begin{array}{c}
\frac{\delta H_{2}}{\delta v} \\
\frac{\delta H_{2}}{\delta w}
\end{array}\right)=B_{2}\left(\begin{array}{c}
\frac{\delta H_{1}}{\delta v} \\
\frac{\delta H_{1}}{\delta w}
\end{array}\right)=\left(\begin{array}{c}
2 w_{x} \\
-\frac{2}{3} v v_{x}-\frac{1}{6} w_{x x x}
\end{array}\right),
$$


where

$$
\begin{gathered}
B_{1}=\left(\begin{array}{cc}
0 & \partial \\
\partial & 0
\end{array}\right), \\
B_{2}=\frac{1}{3}\left(\begin{array}{cc}
2 \partial^{3}+2 v \partial+v_{x} & 3 w \partial+2 w_{x} \\
3 w \partial+w_{x} & -\frac{1}{6}\left(\partial^{5}+5 v \partial^{3}+\frac{15}{2} v_{x} \partial^{2}+\frac{9}{2} v_{x x} \partial+4 v^{2} \partial+v_{x x x}+4 v v_{x}\right)
\end{array}\right)
\end{gathered}
$$

are the two standard Hamiltonian operators of the Boussinesq equation and

$$
H_{1}=\int w d x, H_{2}=\int\left(\frac{1}{12} v_{x}^{2}-\frac{1}{9} v^{3}+w^{2}\right) d x .
$$

For $N$ distinct real eigenvalues $\lambda_{j}$, consider the following spectral problem and its adjoint spectral problem

$$
\begin{aligned}
\varphi_{j x x x}+v \varphi_{j x}+\left(\frac{1}{2} v_{x}+w\right) \varphi_{j} & =\lambda \varphi_{j}, \\
\varphi_{j x x x}^{*}+v \varphi_{j x}^{*}+\left(\frac{1}{2} v_{x}-w\right) \varphi_{j}^{*} & =-\lambda \varphi_{j}^{*}, j=1,2, \cdots, N .
\end{aligned}
$$

We have

$$
\frac{\delta \lambda_{j}}{\delta v}=\frac{3}{2}\left(\varphi_{j x} \varphi_{j}^{*}-\varphi_{j} \varphi_{j x}^{*}\right), \frac{\delta \lambda_{j}}{\delta w}=3 \varphi_{j} \varphi_{j}^{*}
$$

The generalized Kupershmidt deformed Boussinesq equation is given by (9) with $\alpha_{j}=\lambda_{j}$ as follows

$$
\begin{gathered}
\left(\begin{array}{c}
v \\
w
\end{array}\right)_{t}=B_{1}\left(\left(\begin{array}{c}
\frac{\delta H_{2}}{\delta v} \\
\frac{\delta H_{2}}{\delta w}
\end{array}\right)-\sum_{j=1}^{N}\left(\begin{array}{c}
\frac{\delta \lambda_{j}}{\delta v} \\
\frac{\delta \lambda_{j}}{\delta w}
\end{array}\right)\right), \\
\left(B_{2}-\lambda_{j} B_{1}\right)\left(\begin{array}{c}
\frac{\delta \lambda_{j}}{\delta v} \\
\frac{\delta \lambda_{j}}{\delta w}
\end{array}\right)=0, j=1,2, \cdots, N .
\end{gathered}
$$

Set

$$
\begin{gathered}
f_{j}=\varphi_{j x x x}+v \varphi_{j x}+\left(\frac{1}{2} v_{x}+w\right) \varphi_{j}-\lambda_{j} \varphi_{j}, \\
\left(B_{2}-\lambda_{j} B_{1}\right)\left(\begin{array}{c}
\frac{\delta \lambda_{j}}{\delta v} \\
\frac{\delta \lambda_{j}}{\delta w}
\end{array}\right)=\left(\begin{array}{c}
\mathscr{A}_{j} \\
\mathscr{B}_{j}
\end{array}\right), j=1,2, \cdots, N .
\end{gathered}
$$


Direct calculation gives

$$
\mathscr{A}_{j}=\varphi_{j}^{*} f_{j x}+2 \varphi_{j x}^{*} f_{j}-\left(2 \varphi_{j x}^{*} f_{j}+\varphi_{j}^{*} f_{j x}\right)^{*},
$$

which leads to

$$
f_{j}=\frac{\mu_{j}}{\varphi_{j}^{* 2}}, \quad f_{j}^{*}=-\frac{\mu_{j}}{\varphi_{j}^{2}} .
$$

Using (26b), we get

$$
\begin{aligned}
\mathscr{B}_{j}= & -\frac{1}{3}\left(2 v \varphi_{j}+5 \varphi_{j x x}\right)\left(f_{j}^{*}+\frac{\mu_{j}}{\varphi_{j}^{2}}\right)-\frac{1}{3}\left(2 v \varphi_{j}^{*}+5 \varphi_{j x x}^{*}\right)\left(f_{j}-\frac{\mu_{j}}{\varphi_{j}^{* 2}}\right)-\frac{5}{6} \varphi_{j x}^{*}\left(f_{j}-\frac{\mu_{j}}{\varphi_{j}^{* 2}}\right)_{x} \\
- & \frac{5}{6} \varphi_{j x}\left(f_{j}^{*}+\frac{\mu_{j}}{\varphi_{j}^{2}}\right)_{x}-\frac{1}{6} \varphi_{j}^{*}\left(f_{j}-\frac{\mu_{j}}{\varphi_{j}^{* 2}}\right)_{x x}-\frac{1}{6} \varphi_{j}\left(f_{j}^{*}+\frac{\mu_{j}}{\varphi_{j}^{2}}\right)_{x x}+\frac{2 \mu_{j}}{3 \varphi_{j}^{3} \varphi_{j}^{* 3}} \\
& {\left[v \varphi_{j}^{2} \varphi_{j}^{* 2}\left(\varphi_{j}^{*}-\varphi_{j}\right)-\varphi_{j}^{* 3} \varphi_{j x}^{2}+\varphi_{j}^{3}\left(\varphi_{j x}^{* 2}-2 \varphi_{j}^{*} \varphi_{j x x}^{*}\right)+2 \varphi_{j}^{* 3} \varphi_{j} \varphi_{j x x}\right] } \\
= & \frac{2 \mu_{j}}{3 \varphi_{j}^{3} \varphi_{j}^{* 3}}\left[v \varphi_{j}^{2} \varphi_{j}^{* 2}\left(\varphi_{j}^{*}-\varphi_{j}\right)-\varphi_{j}^{* 3} \varphi_{j x}^{2}+\varphi_{j}^{3}\left(\varphi_{j x}^{* 2}-2 \varphi_{j}^{*} \varphi_{j x x}^{*}\right)+2 \varphi_{j}^{* 3} \varphi_{j} \varphi_{j x x}\right], \quad \text { (27) }
\end{aligned}
$$

which yields to $\mu_{j}=0$. Thus, the generalized Kupershmidt deformed Boussinesq equation (25) gives the following integrable system

$$
\begin{aligned}
& v_{t}=2 w_{x}-3 \sum_{j=1}^{N}\left(\varphi_{j} \varphi_{j}^{*}\right)_{x}, \\
& w_{t}=-\frac{1}{6}\left(4 v v_{x}+v_{x x x}\right)-\frac{3}{2}\left(\varphi_{j x x} \varphi_{j}^{*}-\varphi_{j} \varphi_{j x x}^{*}\right), \\
& \varphi_{j x x x}+v \varphi_{j x}+\left(\frac{1}{2} v_{x}+w\right) \varphi_{j}=\lambda_{j} \varphi_{j}, \\
& \varphi_{j x x x}^{*}+v \varphi_{j x}^{*}+\left(\frac{1}{2} v_{x}-w\right) \varphi_{j}^{*}=-\lambda_{j} \varphi_{j}^{*}, j=1,2, \cdots, N
\end{aligned}
$$

which just is the Boussinesq equation with self-consistent sources and has the following Lax representation 26

$$
\begin{aligned}
& L_{t}=\left[\partial^{2}+\frac{2}{3} v+\sum_{j=1}^{N} \varphi_{j} \partial^{-1} \varphi_{j}^{*}, L\right] \\
& L \psi=\left(\partial^{3}+v \partial+\frac{1}{2} v_{x}+w\right) \psi=\lambda \psi, \\
& \psi_{t}=\left(\partial^{2}+\frac{2}{3} v+\sum_{j=1}^{N} \varphi_{j} \partial^{-1} \varphi_{j}^{*}\right) \psi .
\end{aligned}
$$




\section{The generalized Kupershmidt deformation of Jaulent-Miodek equation and its bi-Hamiltonian structure}

\subsection{The generalized Kupershmidt deformation of Jaulent- Miodek equation}

The JM eigenvalue problem reads [27]

$$
\left(\begin{array}{l}
\psi_{1} \\
\psi_{2}
\end{array}\right)_{x}=U\left(\begin{array}{l}
\psi_{1} \\
\psi_{2}
\end{array}\right), U=\left(\begin{array}{cc}
0 & 1 \\
-\lambda^{2}+\lambda q+r & 0
\end{array}\right),
$$

the associated JM hierarchy is

$$
\left(\begin{array}{l}
q \\
r
\end{array}\right)_{t_{n}}=B_{1}\left(\begin{array}{l}
b_{n+2} \\
b_{n+1}
\end{array}\right)=B_{1}\left(\begin{array}{c}
\frac{\delta H_{n+1}}{\delta q} \\
\frac{\delta H_{n+1}}{\delta r}
\end{array}\right)=B_{2}\left(\begin{array}{c}
\frac{\delta H_{n}}{\delta q} \\
\frac{\delta H_{n}}{\delta r}
\end{array}\right)
$$

where

$$
\begin{gathered}
B_{1}=\left(\begin{array}{cc}
0 & 2 \partial \\
2 \partial & -q_{x}-2 q \partial
\end{array}\right), B_{2}=\left(\begin{array}{cc}
2 \partial & 0 \\
0 & r_{x}+2 r \partial-\frac{1}{2} \partial^{3}
\end{array}\right), \\
\left(\begin{array}{c}
b_{n+2} \\
b_{n+1}
\end{array}\right)=L\left(\begin{array}{c}
b_{n+1} \\
b_{n}
\end{array}\right), n=1,2, \cdots \\
b_{0}=b_{1}=0, b_{2}=-1, H_{n}=\frac{1}{n-1}\left(2 b_{n+2}-q b_{n+1}\right) .
\end{gathered}
$$

For $N$ distinct real eigenvalues $\lambda_{j}$, from the spectral problem

$$
\varphi_{1 j x}=\varphi_{2 j}, \varphi_{2 j x}=\left(-\lambda_{j}^{2}+\lambda_{j} q+r\right) \varphi_{1 j}
$$

we have

$$
\frac{\delta \lambda_{j}}{\delta q}=\frac{1}{2} \lambda_{j} \varphi_{1 j}^{2}, \frac{\delta \lambda_{j}}{\delta r}=\frac{1}{2} \varphi_{1 j}^{2} .
$$


Similarly, the generalized Kupershmidt deformation (9) with $\alpha_{j}=\lambda_{j}$ for JM hierarchy gives rise to

$$
\begin{aligned}
& \left(\begin{array}{c}
q \\
r
\end{array}\right)_{t_{n}}=B_{1}\left(\left(\begin{array}{c}
\frac{\delta H_{n+1}}{\delta q} \\
\frac{\delta H_{n+1}}{\delta r}
\end{array}\right)+\sum_{j=1}^{N}\left(\begin{array}{c}
\frac{\delta \lambda_{j}}{\delta q} \\
\frac{\delta \lambda_{j}}{\delta r}
\end{array}\right)\right), \\
& \left(B_{2}-\lambda_{j} B_{1}\right)\left(\begin{array}{c}
\frac{\delta \lambda_{j}}{\delta q} \\
\frac{\delta \lambda_{j}}{\delta r}
\end{array}\right)=0, j=1,2, \cdots, N .
\end{aligned}
$$

The first equation in (31b) is an identity and second one in (31b) yields

$\varphi_{1 j}\left(\varphi_{1 j x x}-r \varphi_{1 j}-\lambda_{j} q \varphi_{1 j}+\lambda_{j}^{2} \varphi_{1 j}\right)_{x}+3 \varphi_{1 j x}\left(\varphi_{1 j x x}-r \varphi_{1 j}-\lambda_{j} q \varphi_{1 j}+\lambda_{j}^{2} \varphi_{1 j}\right)=0$

which, by setting $\varphi_{2 j}=\varphi_{1 j x}$, leads to

$$
\varphi_{2 j x}=\left(-\lambda_{j}^{2}+\lambda_{j} q+r\right) \varphi_{1 j}+\frac{\mu_{j}}{\varphi_{1 j}^{3}}, j=1,2, \cdots, N .
$$

Then the generalized Kupershmidt deformation with of JM equation (31) with $n=3$ gives rise to the following integrable system

$$
\begin{aligned}
& q_{t}=-r_{x}-\frac{3}{2} q q_{x}+2 \sum_{j=1}^{N} \varphi_{1 j} \varphi_{2 j}, \\
& r_{t}=\frac{1}{4} q_{x x x}-q_{x} r-\frac{1}{2} q r_{x}+\sum_{j=1}^{N}\left[2\left(\lambda_{j}-q\right) \varphi_{1 j} \varphi_{2 j}-\frac{1}{2} q_{x} \varphi_{1 j}^{2}\right], \\
& \varphi_{1 j x}=\varphi_{2 j}, \varphi_{2 j x}=\left(-\lambda_{j}^{2}+\lambda_{j} q+r\right) \varphi_{1 j}+\frac{\mu_{j}}{\varphi_{1 j}^{3}} j=1,2, \cdots, N
\end{aligned}
$$

which just is the integrable RD-JMESCS and has the Lax representation (14a) with [15]

$$
\begin{gathered}
U=\left(\begin{array}{cc}
0 & 1 \\
-\lambda^{2}+\lambda q+r & 0
\end{array}\right), \\
V=\left(\begin{array}{cc}
\frac{1}{4} q_{x} & -\lambda-\frac{1}{2} q \\
\lambda^{3}-\frac{1}{2} q \lambda^{2}-\left(\frac{1}{2} q^{2}+r\right) \lambda+\frac{1}{4} q_{x x}-\frac{1}{2} q r & -\frac{1}{4} q_{x}
\end{array}\right) \\
+\frac{1}{2}\left(\begin{array}{cc}
0 & 0 \\
\lambda\left\langle\Phi_{1}, \Phi_{1}\right\rangle-\left\langle\Lambda \Phi_{1}, \Phi_{1}\right\rangle-q\left\langle\Phi_{1}, \Phi_{1}\right\rangle & 0
\end{array}\right)+\frac{1}{2} \sum_{j=1}^{N} \frac{1}{\lambda-\lambda_{j}}\left(\begin{array}{cc}
\phi_{1 j} \phi_{2 j} & -\phi_{1 j}^{2} \\
\phi_{2 j}^{2}+\frac{\mu_{j}}{\phi_{1 j}^{2}} & -\phi_{1 j} \phi_{2 j}
\end{array}\right)
\end{gathered}
$$




\subsection{The bi-Hamiltonian structure of GKDJME}

In the following, we will show how to construct the bi-Hamiltonian structure for the generalized Kupershmidt deformation of a bi-Hamiltonian system. We follow the method in [17-20] to construct the bi-Hamiltonian formalism with $t-$ type Hamiltonian operator for GKDJME by taking $x$ as the evolution parameter and $t$ as the 'spatial' variable. We denote the inner product in $\mathbb{R}^{N}$ by $\langle.,$.$\rangle and$ $\Phi_{i}=\left(\varphi_{i 1}, \varphi_{i 2}, \cdots, \varphi_{i N}\right)^{T}, i=1,2, \mu=\left(\mu_{1}, \cdots, \mu_{N}\right)^{T}, \Lambda=\operatorname{diag}\left(\lambda_{1}, \cdots, \lambda_{N}\right)$.

Eq.(32) can be written as

$$
\begin{aligned}
& \left(\begin{array}{c}
q \\
r
\end{array}\right)_{t}=B_{1}\left(\begin{array}{c}
\frac{1}{8} q_{x x}-\frac{3}{4} q r-\frac{5}{16} q^{3}+\frac{1}{2}\left\langle\Lambda \Phi_{1}, \Phi_{1}\right\rangle \\
-\frac{1}{2} r-\frac{3}{8} q^{2}+\frac{1}{2}\left\langle\Phi_{1}, \Phi_{1}\right\rangle
\end{array}\right) \\
& \varphi_{1 j x}=\varphi_{2 j}, \varphi_{2 j x}=-\lambda_{j}^{2} \varphi_{1 j}+q \lambda_{j} \varphi_{1 j}+r \varphi_{1 j}+\frac{\mu_{j}}{\varphi_{1 j}^{3}} .
\end{aligned}
$$

Notices that Kernel of $B_{1}$ is $\left(c_{1}+\frac{1}{2} q c_{2}, c_{2}\right)^{T}$, we may rewrite (33a) as

$$
\begin{aligned}
& \frac{1}{8} q_{x x}-\frac{3}{4} q r-\frac{5}{16} q^{3}+\frac{1}{2}\left\langle\Lambda \Phi_{1}, \Phi_{1}\right\rangle=c_{1}+\frac{1}{2} q c_{2},-\frac{1}{2} r-\frac{3}{8} q^{2}+\frac{1}{2}\left\langle\Phi_{1}, \Phi_{1}\right\rangle=c_{2} \\
& c_{1 x}=\frac{1}{2} \partial_{t}\left(r+\frac{1}{4} q^{2}\right), c_{2 x}=\frac{1}{2} \partial_{t} q .
\end{aligned}
$$

By introducing

$$
q_{1}=q, \quad p_{1}=-\frac{1}{8} q_{x}
$$


Eqs. (33b) and (34b) give rise to the t-type Hamiltonian form

$$
R_{x}=G_{1} \frac{\delta F_{1}}{\delta R}
$$

where

$$
\begin{aligned}
& R=\left(\Phi_{1}^{T}, q_{1}, \Phi_{2}^{T}, p_{1}, c_{1}, c_{2}\right)^{T}, \\
& F_{1}=-4 p_{1}^{2}-\frac{1}{16} q_{1}^{4}-\frac{1}{2} q_{1}^{2} c_{2}+q_{1} c_{1}-c_{2}^{2}+\frac{3}{8} q_{1}^{2}\left\langle\Phi_{1}, \Phi_{1}\right\rangle-\frac{1}{2} q_{1}\left\langle\Lambda \Phi_{1}, \Phi_{1}\right\rangle \\
& +\frac{1}{2}\left\langle\Phi_{2}, \Phi_{2}\right\rangle+\frac{1}{2}\left\langle\Lambda^{2} \Phi_{1}, \Phi_{1}\right\rangle+c_{2}\left\langle\Phi_{1}, \Phi_{1}\right\rangle-\frac{1}{4} \sum_{j=1}^{N} \varphi_{1 j}^{4}+\frac{1}{2} \sum_{j=1}^{N} \frac{\mu_{j}}{\varphi_{1 j}^{2}},
\end{aligned}
$$

and the $t$-type Hamiltonian operator $G_{1}$ is given by

$$
G_{1}=\left(\begin{array}{cccc}
0 & I_{(N+1) \times(N+1)} & 0 & 0 \\
-I_{(N+1) \times(N+1)} & 0 & 0 & 0 \\
0 & 0 & 0 & \frac{1}{2} \partial_{t} \\
0 & 0 & \frac{1}{2} \partial_{t} & 0
\end{array}\right) .
$$

The modified Jaulent-Miodek (MJM) eigenvalue problem reads 28]

$$
\left(\begin{array}{c}
\tilde{\psi}_{1} \\
\tilde{\psi}_{2}
\end{array}\right)_{x}=\tilde{U}(\tilde{u}, \lambda)\left(\begin{array}{c}
\tilde{\psi}_{1} \\
\tilde{\psi}_{2}
\end{array}\right), \quad \tilde{U}=\left(\begin{array}{cc}
-\tilde{r} & \lambda \\
-\lambda+\tilde{q} & \tilde{r}
\end{array}\right), \tilde{u}=\left(\begin{array}{c}
\tilde{r} \\
\tilde{q}
\end{array}\right)
$$

the associated MJM equation is of the form

$$
\tilde{u}_{t}=\left(\begin{array}{c}
\tilde{r} \\
\tilde{q}
\end{array}\right)_{t}=\left(\begin{array}{c}
-\frac{1}{4} \tilde{q}_{x x}-\frac{1}{2}(\tilde{q} \tilde{r})_{x} \\
-2 \tilde{r} \tilde{r}_{x}-\frac{3}{2} \tilde{q} \tilde{q}_{x}+\tilde{r}_{x x}
\end{array}\right)=\tilde{B}_{1} \frac{\delta \tilde{H}_{2}}{\delta \tilde{u}}
$$

where $\tilde{B}_{1}=\left(\begin{array}{cc}\frac{1}{2} \partial & 0 \\ 0 & 2 \partial\end{array}\right), \tilde{H}_{2}=-\frac{1}{2} \tilde{q}_{x} \tilde{r}-\frac{1}{2} \tilde{q} \tilde{r}^{2}-\frac{1}{8} \tilde{q}^{3}$.

We have

$$
\frac{\delta \lambda}{\delta \tilde{u}}=\left(\begin{array}{c}
\tilde{\varphi}_{1} \tilde{\varphi}_{2} \\
\frac{1}{2} \tilde{\varphi}_{1}^{2}
\end{array}\right)
$$

Then the Rosochatius deformation of MJM equation with self-consistent sources 
(RD-MJMSCS) is defined as

$$
\begin{aligned}
& \left(\begin{array}{c}
\tilde{r} \\
\tilde{q}
\end{array}\right)_{t}=\tilde{B}_{1}\left(\frac{\delta \tilde{H}_{2}}{\delta \tilde{u}}+\frac{\delta \lambda}{\delta \tilde{u}}\right)=\tilde{B}_{1}\left(\begin{array}{c}
-\frac{1}{2} \tilde{q}_{x}-\tilde{q} \tilde{r}+\left\langle\tilde{\Phi}_{1}, \tilde{\Phi}_{2}\right\rangle \\
-\frac{1}{2} \tilde{r}^{2}-\frac{3}{8} \tilde{q}^{2}+\frac{1}{2} \tilde{r}_{x}+\frac{1}{2}\left\langle\tilde{\Phi}_{1}, \tilde{\Phi}_{1}\right\rangle
\end{array}\right) \\
& \tilde{\varphi}_{1 j x}=-\tilde{r} \tilde{\varphi}_{1 j}+\lambda_{j} \tilde{\varphi}_{2 j}, \tilde{\varphi}_{2 j x}=-\lambda_{j} \tilde{\varphi}_{1 j}+\tilde{q} \tilde{\varphi}_{1 j}+\tilde{r} \tilde{\varphi}_{2 j}+\frac{\mu_{j}}{\lambda_{j} \tilde{\varphi}_{1 j}^{3} .}
\end{aligned}
$$

Since the Kernel of $\tilde{B}_{1}$ is $\left(\tilde{c}_{1}, \tilde{c}_{2}\right)^{T}$, let

$$
\begin{gathered}
-\frac{1}{2} \tilde{q}_{x}-\tilde{q} \tilde{r}+\left\langle\tilde{\Phi}_{1}, \tilde{\Phi}_{2}\right\rangle=\tilde{c}_{1}, \quad-\frac{1}{2} \tilde{r}^{2}-\frac{3}{8} \tilde{q}^{2}+\frac{1}{2} \tilde{r}_{x}+\frac{1}{2}\left\langle\tilde{\Phi}_{1}, \tilde{\Phi}_{1}\right\rangle=\tilde{c}_{2}, \\
\tilde{q}_{1}=\tilde{q}, \tilde{p}_{1}=-\frac{1}{2} \tilde{r}, \tilde{R}=\left(\tilde{\Phi}_{1}^{T}, \tilde{q}_{1}, \tilde{\Phi}_{2}^{T}, \tilde{p}_{1}, \tilde{c}_{1}, \tilde{c}_{2}\right)^{T},
\end{gathered}
$$

then RD-MJMSCS (38) can be written as a t-type Hamiltonian system

$$
\tilde{R}_{x}=\tilde{G}_{1} \frac{\delta \tilde{F}_{1}}{\delta \tilde{R}}
$$

where

$$
\begin{aligned}
\tilde{F}_{1}= & -2 \tilde{p}_{1} \tilde{c}_{1}+\tilde{q}_{1} \tilde{c}_{2}+2 \tilde{p}_{1}^{2} \tilde{q}_{1}+\frac{1}{8} \tilde{q}_{1}^{3}+2 \tilde{p}_{1}\left\langle\tilde{\Phi}_{1}, \tilde{\Phi}_{2}\right\rangle-\frac{1}{2} \tilde{q}_{1}\left\langle\tilde{\Phi}_{1}, \tilde{\Phi}_{1}\right\rangle \\
& +\frac{1}{2}\left\langle\Lambda \tilde{\Phi}_{2}, \tilde{\Phi}_{2}\right\rangle+\frac{1}{2}\left\langle\Lambda \tilde{\Phi}_{1}, \tilde{\Phi}_{1}\right\rangle+\sum_{j=1}^{N} \frac{\mu_{j}}{2 \lambda_{j} \tilde{\varphi}_{1 j}^{2}}, \\
G_{1}= & \left(\begin{array}{cccc}
0 & I_{(N+1) \times(N+1)} & 0 & 0 \\
-I_{(N+1) \times(N+1)} & 0 & 0 & 0 \\
0 & 0 & 2 \partial_{t} & 0 \\
0 & 0 & 0 & \frac{1}{2} \partial_{t}
\end{array}\right)
\end{aligned}
$$

The Miura map relating systems (36) and (39), i.e. $R=M(\tilde{R})$, is given by

$$
\begin{aligned}
& \Phi_{1}=\tilde{\Phi}_{1}, \Phi_{2}=\Lambda \tilde{\Phi}_{2}+2 \tilde{p}_{1} \tilde{\Phi}_{1}, \\
& q_{1}=\tilde{q}_{1}, p_{1}=-\frac{1}{2} \tilde{q}_{1} \tilde{p}_{1}-\frac{1}{4}\left\langle\tilde{\Phi}_{1}, \tilde{\Phi}_{2}\right\rangle+\frac{1}{4} \tilde{c}_{1}, \\
& c_{1}=\frac{1}{2} \tilde{F}_{1}+\partial_{t} \tilde{p}_{1}, \quad c_{2}=\tilde{c}_{2} .
\end{aligned}
$$

Denote

$$
M^{\prime} \equiv \frac{D R}{D \tilde{R}^{T}}
$$


where $\frac{D R}{D \tilde{R}^{T}}$ is the Jacobi matrix consisting of Frechet derivative of $M, M^{\prime}$ * denotes adjoint of $M^{\prime}$. According to the standard procedure 29], applying the map $M$ (40) to the first Hamiltonian structure of Eq.(39), we can generates the second Hamiltonian structure of Eq.(36)

$$
G_{2}=M \tilde{G}_{1} M^{*}=\left(\begin{array}{cccccc}
0 & 0 & \Lambda & -\frac{1}{4} \Phi_{1} & \frac{1}{2} \Phi_{2} & 0 \\
0 & 0 & 2 \Phi_{1}^{T} & -\frac{1}{2} q_{1} & -4 p_{1}-\partial_{t} & 0 \\
-\Lambda & 2 \Phi_{1} & 0 & \frac{1}{4} \Phi_{2} & g_{35} & 0 \\
\frac{1}{4} \Phi_{1}^{T} & \frac{1}{2} q_{1} & -\frac{1}{4} \Phi_{2}^{T} & \frac{1}{8} \partial_{t} & g_{45} & 0 \\
-\frac{1}{2} \Phi_{2}^{T} & 4 p_{1}-\partial_{t} & -g_{35} & -g_{45} & g_{55} & \partial_{t} q_{1} \\
0 & 0 & 0 & 0 & q_{1} \partial_{t} & 2 \partial_{t}
\end{array}\right)
$$

where

$$
\begin{gathered}
g_{35}=\frac{1}{2} q_{1} \Lambda \Phi_{1}-\frac{1}{2} \Lambda^{2} \Phi_{1}-\frac{3}{8} q_{1}^{2} \Phi_{1}-c_{2} \Phi_{1}+\frac{1}{4} \Phi_{1}\left\langle\Phi_{1}, \Phi_{1}\right\rangle+\left(\frac{\mu_{1}}{\varphi_{1 j}^{3}}, \cdots, \frac{\mu_{N}}{\varphi_{1 N}^{3}}\right)^{T} \\
g_{45}=-\frac{1}{2} c_{1}+\frac{1}{4}\left\langle\Lambda \Phi_{1}, \Phi_{1}\right\rangle-\frac{3}{8} q_{1}\left\langle\Phi_{1}, \Phi_{1}\right\rangle+\frac{1}{2} q_{1} c_{2}+\frac{1}{8} q_{1}^{3} \\
g_{55}=\partial_{t}\left(\frac{1}{4}\left\langle\Phi_{1}, \Phi_{1}\right\rangle-\frac{1}{2} c_{2}\right)+\left(\frac{1}{4}\left\langle\Phi_{1}, \Phi_{1}\right\rangle-\frac{1}{2} c_{2}\right) \partial_{t}-\frac{1}{4} q_{1} \partial_{t} q_{1} .
\end{gathered}
$$

Thus we get the bi-Hamiltonian structure for Eq. (36a)-(36c)

$$
R_{x}=G_{1} \frac{\delta F_{1}}{\delta R}=G_{2} \frac{\delta F_{0}}{\delta R}, \quad F_{0}=2 c_{1} .
$$

\section{Conclusion}

It is known that there were some methods to construct a new integrable system starting from a bi-Hamiltonian system. The main purpose of this paper is to propose the generalized Kupershmidt deformation (GKD) of bi-Hamiltonian systems to construct new systems from integrable bi-Hamiltonian systems which is conjectured to be integrable. We have not be able to prove the integrability of the generalized Kupershmidt deformation of bi-Hamiltonian systems in general. However, for many specific cases, such as for KdV equation, Boussinesq 
equation, Jaulent-Miodek equation and Camassa-Holm equation, by using this generalized Kupershmidt deformation some new integrable systems are derived from integrable bi-Hamiltonian systems. We present a general procedure to convert this generalized Kupershmidt deformation of the bi-Hamiltonian systems into an integrable Rosochatius deformation of soliton equation with selfconsistent sources, as well as to transform it into a $t$-type bi-Hamiltonian system. These imply that the generalized Kupershmidt deformation of bi-Hamiltonian systems provides a way to construct new integrable system from an integrable bi-Hamiltonian systems. On other hand the generalized Kupershmidt deformation of bi-Hamiltonian systems also offers a new method to obtain the integrable Rosochatius deformation of soliton equation with self-consistent sources, which is quite different from the method used before. In the further we will continue to study the integrability of the generalized Kupershmidt deformation of biHamiltonian systems in general. We believe that the method in [6] is helpful for proving the bi-Hamiltonian structure of the generalized Kupershmidt deformation.

\section{Acknowledgement}

This work is supported by National Basic Research Program of China (973 Program) (2007CB814800), National Natural Science Foundation of China $(10801083,10901090)$ and Chinese Universities Scientific Fund (2009JS42,2009-2-05).

\section{References}

[1] B. Fuchssteiner and A. S. Fokas, Physica 4D 47 (1981).

[2] Peter J. Olver and Philip Rosenau, Phys. Rev. E 531900 (1996). 
[3] A. Karasu-Kalkani, A. Karasu, A. Sakovich, S. Sakovich and R. Turhan, J. Math. Phys. 49073516 (2008).

[4] Boris A. Kupershmidt, Phys. Lett. A 3722634 (2008).

[5] Y. Q. Yao and Y. B. Zeng, Lett. Math. Phys. 86193 (2008).

[6] P. H. M. Kersten, I. S. Krasil'shchik, A. M. Verbovetsky and R. Vitolo, Acta. Appl. Math. 10975 (2010).

[7] E. Rosochatius, dissertation, University of Götingen, 1877.

[8] S. Wojciechowski, Phys. Scr.34 304 (1986).

[9] R. Kubo, W. Ogura, T. Saito and Y. Yasui, Phys. Lett. A 2516 (1999).

[10] R. G. Zhou, J. Math. Phys. 48103510 (2007).

[11] C. Bartocci, G. Falqui and M. Pedroni, Diff. Geom. Applic. 21349 (2004).

[12] M. Kruczenski, J. G. Russo and A. A. Tseytlin, J. High Energy Phys. 10 063 (2006).

[13] P. L. Christiansen, J. C. Eilbeck, V. Z. Enolskii and N. A. Kostov, Proceedings: Mathematical and Physical Sciences.451 685 (1995).

[14] Y. Q. Yao and Y. B. Zeng, J. Phys. A: Math. Theor. 41295205 (2008) .

[15] Y. Q. Yao and Y. B. Zeng, Commun. Theor. Phys. 52193 (2009).

[16] V. I. Arnold, Mathematical Methods of Classical Mechanics, SprigerVerlag, New York (1978).

[17] B. Fuchssteiner, W. Oevel, Physica A 14567 (1987).

[18] A. P. Fordy, Physica D 8720 (1995).

[19] M. Blaszak, J. Math. Phys. 364826 (1995). 
[20] Y. B. Zeng, Physica A 262405 (1999).

[21] V. K. Menlikov, Inverse Probl. 6233 (1990).

[22] R. Camassa and D. Holm, Phys. Rev. Lett. 711661 (1993).

[23] A. Parker, Proc. R. Soc. Lond. A 4602929 (2004).

[24] Y. H. Huang, Y. Q. Yao and Y. B. Zeng., Commun. Theor. Phys. 53403 (2010).

[25] A. P. Fordy and J. Gibbons, J. Math. Phys. 221170 (1981).

[26] Y. B. Zeng, Acta Mathematica Scientia 1797 (1997).

[27] M. Jaulent and K. Miodek. Lett. Math. Phys. 1243 (1976).

[28] A. P. Fordy, Isospectral flows: their Hamiltonian structure, Miura maps and master symmetries, in: P. J. Olver, D. H. Sattinger(Eds.),Proc. IWA Workshop on Applications of Solitons, 1991, preprint.

[29] B. A. Kupershmidt and J. Wilson, Invent Math.62 403 (1981). 